\title{
Effect of Carbon Dioxide on the Properties and Chemical Composition of Bentonite in Aqueous Suspension
}

\author{
Mezhidov V. Kh. \\ SICC "Nanotechnologies and Nanomaterials" \\ Grozny State Oil Technical University. \\ Grozny, Russia \\ nizkp@mail.ru \\ Gatsaev Z.Sh. \\ SICC "Nanotechnologies and Nanomaterials" \\ Grozny State Oil Technical University \\ Grozny, Russia \\ nizkp@mail.ru
}

\author{
Dadashev R.H. \\ Laboratory of Theoretical and Experimental Physics \\ Research Institute named H.I. Ibragimova of RAS \\ Grozny, Russia \\ raykom50@mail.ru.ru
}

\author{
Gayrabekov I.G \\ Department of "Geodesy and land cadastre" \\ Grozny State Oil Technical University \\ Grozny, Russia \\ ibragim.ggni@mail.ru
}

\begin{abstract}
In the process of using drilling muds in the oil and gas reservoirs, bentonite clay accumulates, which precludes their development, if not swelling it with carbon dioxide. The objection of this paper is to present the results of the sedimentation study and the degree of bentonite swelling depending on time for suspensions with different water concentrations of solid phase and carbon dioxide. If carbon dioxide gas is introduced into the dispersion medium, $0.12 \%$ of the weight, sedimentation is completed within the first 2-3 hours with a lower value of swelling degree. Precipitates obtained in samples with gas have particle sizes and chemical composition significantly different from the reference variant. It is found that in suspensions of bentonite with carbon dioxide the concentration of silicon in the sediment decreases to $10 \%$, which is explained by its transfer to the dispersion medium in amorphous form.
\end{abstract}

Keywords - bentonite, sedimentation, swelling, carbonated dispertion medium, particles' sizes

\section{INTRODUCTION}

Bentonite has a remarkable ability to swell when interacting with water, increasing its volume up to 15 times. This allows to find gels with thixotropic properties with low concentrations of bentonite. Such gels are the basis for the production of drilling muds with the help of reagents that regulate sedimentation stability, viscosity, density, dispersion of the solid phase [1-4]. Drilling muds make drilled sludge, create a layer along the well that protects it from groundwater, and acts as a lubricant. However, in the majority of productive oil and gas reservoirs, a certain amount of clay accumulates, which is also brought into the well bottom zone along with the mud filtrate when drilling the layer, overhaul and current maintenance. This clay is able to swell and significantly reduce oil recovery. Sometimes such swelling excludes the development of oil reservoirs [5]. Therefore, a search aims at salts, acids, surfactants, solutions, gases, allowing to regulate the swelling of clays. Carbon dioxide has such properties, it is pumped into the reservoir and oil recovery is increased, but it is not always economically justified for a selected field [6]. In this work, the effect of carbon dioxide on the degree of swelling of bentonite clay is under research from the Katayama deposit (Chechen Republic), its chemical composition, sedimentation stability of suspensions.

\section{METHODS AND MATERIALS}

In the experiments, activated bentonite in $50 \mathrm{ml}$ graduated plastic ampoules with lids ensuring tightness was used. In each pair of ampoules numbered from 1 to $10,1,2,3,5$, and 7 $\mathrm{g}$ of powdered bentonite were added. Distilled water was added to the ampoules with even numbers to the bentonite and shaken for $3 \mathrm{~min}$. To obtain samples of suspensions with a concentration of bentonite in $\%$ by weight: $1.2 ; 2.4 ; 4.8 ; 7.0$; 11.1. Distilled water enriched with aerated Kubai carbonated water was introduced into odd ampoules. The concentration of carbon dioxide in the samples was determined by two methods: an INS-210 device for measuring $\mathrm{CO}_{2}$ and titration using a $0.1 \mathrm{~N}$ solution of sodium hydroxide and $1 \%$ alcohol solution of phenolphthalein. It was $0.12 \%$ by weight. Possible errors due to the evaporation of carbon dioxide from water into the atmosphere were reduced, reducing its temperature to $3-5{ }^{\circ} \mathrm{C}$, and reducing the time of contact with the environment. Thus, we had 5 reference samples of suspensions with the above concentration of bentonite, having even numbers, and five test samples with odd numbers, differing only in the fact that $0.12 \%$ of carbon dioxide was added to their dispersion medium, were obtained. After 3 min of shaking, the closed samples of the suspensions were placed in a cassette at a temperature of $25^{\circ} \mathrm{C}$, and changes in the volume of precipitation were recorded as a function of time. After $24 \mathrm{~h}$, the $\mathrm{pH}$ of the dispersion medium and the chemical composition of the precipitates dried at $110{ }^{\circ} \mathrm{C}$ were determined. 
Grinding of bentonite was made in a ball mill RM 100 (Germany). The hydrogen indicator of the dispersion medium was determined using a PH-150 MI device. The analyzer of submicron particles HORIBA LD-550 (Japan) investigated the sizes of the particles of bentonite in suspension. Changes in the chemical composition of bentonite were investigated with a QUANTA 3D 200i (USA) scanning electron microscope (SEM) with an integrated system of energy - dispersive microanalysis (EDAX). The degree of swelling of bentonite was determined by the formula: $S=\left(V_{\mathrm{n}}-V_{\mathrm{t}}\right) / V_{\mathrm{t}}$, where $V_{\mathrm{t}}$ is the volume of a solid sample of bentonite before swelling, and $V_{\mathrm{n}}$ - after its swelling.

\section{RESULTS}

The Fig. Figure 1 shows the graphs of the change in the precipitation volume depending on time and concentration of the solid phase (Sat) in bentonite suspensions with even numbers, where the dispersion medium is distilled water.

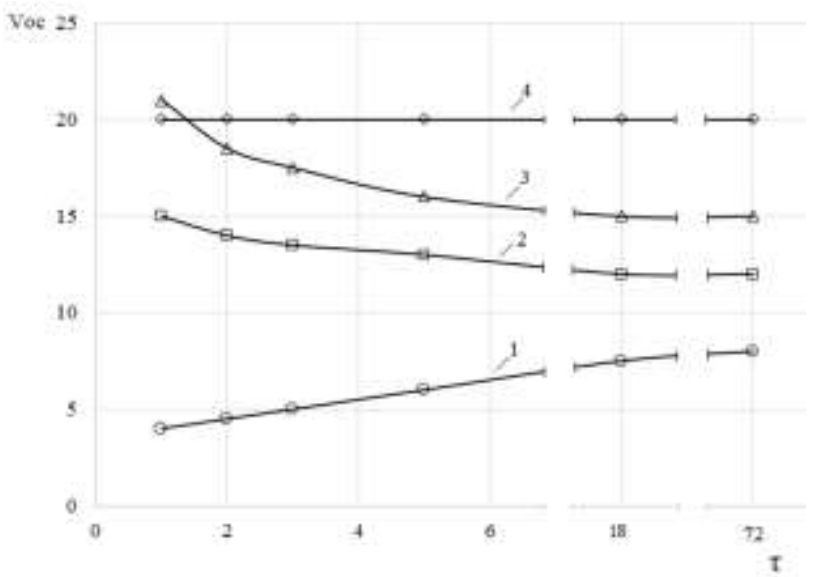

Fig. 1. Dependence of precipitation volume on sedimentation time in suspensions with distilled water at concentrations of bentonite in wt. \%: 1-2.4; $2-4.8 ; 3-7.0 ; 4-11.1$

It is clear that the nature of the change in the sediment volume in a suspension of bentonite significantly depends on its concentration. At low concentrations of bentonite, its particles dispersed with distilled water have dimensions of hundreds of nanometers. They precipitate slowly and after a long time give a high degree of swelling (curve 1). We explain this increase in the degree of swelling as a function of time by the presence in the suspension of a sufficient number of water molecules for the swelling of capillary, adsorption, and osmotic bentonite. An increase in the concentration of bentonite in the suspension alters this dependence, since sedimentation of large particles and their aggregates occurs. In this case, the sediments are compacted; their volumes slowly decrease to a constant value with significantly lower values of the degree of swelling (Fig. 1, curves 2 and 3). At Sat = $11.1 \%$, the volume of sediment of the solid phase practically does not change with time (line 4), since there is not enough water to implement the processes mentioned above, which lead to swelling.

Figure 2 shows the dependencies similar to those presented above, but they were determined on odd samples, that is, in the presence of $0.12 \%$ carbon dioxide in a dispersion medium.
Here, at concentrations of bentonite in suspension up to $4.8 \%$, the change in the volume of sediment is practically zero over time. It is short-term and noticeable when the concentration of bentonite is more than $7 \%$ (Fig. 2, curve 3). It can be seen that in the presence of carbon dioxide, the process of sedimentation of the solid phase in the suspension is completed ten times faster and the formation of precipitates of much smaller volumes occurs. Our experiments show that in suspensions of bentonite with $0.12 \%$ carbon dioxide, particles of bentonite always have a median size of more than a micron.

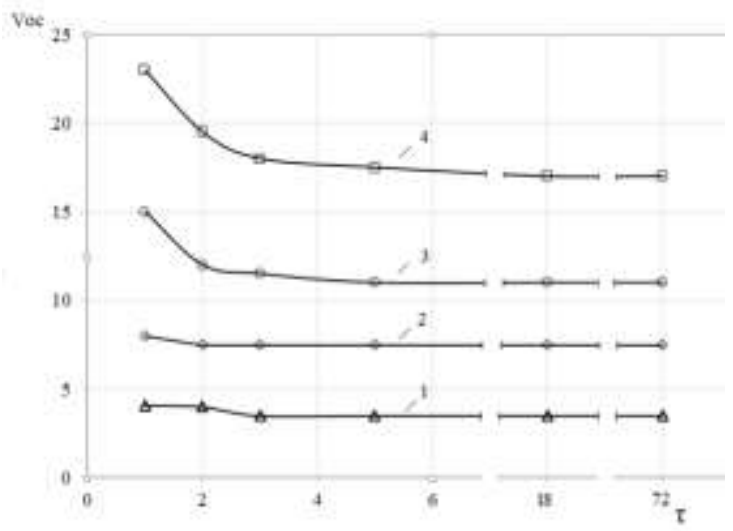

Fig. 2. Dependence of precipitation volume on sedimentation time in suspensions with carbonated distilled water at bentonite concentrations in $\%$ by weight: $1-2.4 ; 2-4.8 ; 3-7.0 ; 4-11.1$

In addition, at high concentrations of bentonite, the nature of changes in precipitation in the presence of carbon dioxide is significant (Fig. 2, curve 4), and in the reference sample (Fig. 1 , graph 4), there is no change in the volume of sediment depending on time. At low concentrations of bentonite, the volume of sediment in the reference sample grows continuously depending on time, and in the experimental one, there is no (curves 1 in Fig. 1 and 2).

Figure 3 shows the dependence of the degree of swelling of bentonite on its concentration in carbonated drinking water Kubai (curve 1), non-carbonated (curve 2) and also in distilled water (curve 3 ).

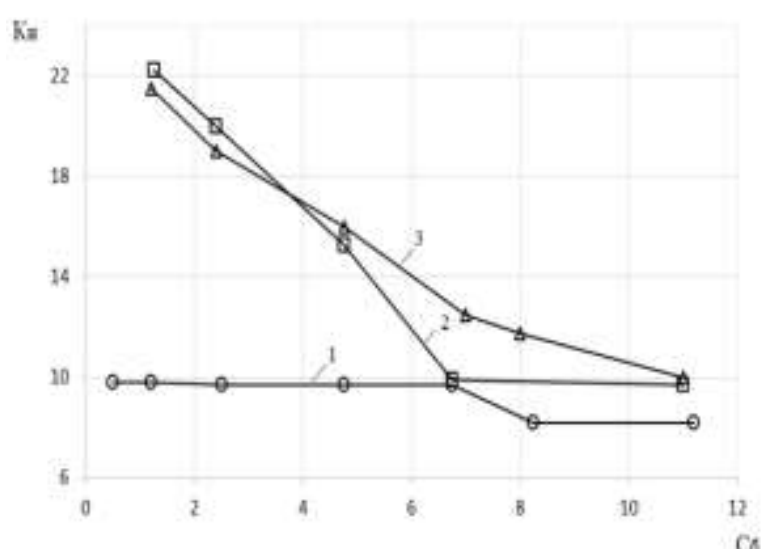

Fig. 3. Dependence of the degree of swelling on the concentration of bentonite (in \% by weight) in distilled water with $\mathrm{CO}_{2}$ gas (curve 1), potable water Kubai (curve 2) and in distilled water (curve 3 ). 
It can be clearly seen that in still water, the degree of bentonite swelling almost do not depend on its concentration, if it is more than $7 \%$. At Sat $=7 \%$, a decrease in the concentration of bentonite to $1.2 \%$ leads to a rapid increase in the degree of swelling to 22 . In water enriched with carbon dioxide for all studied concentrations of bentonite (1.2$11.1 \%$ ), the degree of swelling remains unchanged near 9 units. The degree of swelling of bentonite in distilled water, depending on its concentration, has the same character as for non-carbonated drinking water Kubai, only a little increase in the graph can be noticed.

In our experience, for the suspensions studied the equilibrium value of the $\mathrm{pH}$ of the dispersion medium is reached in the first hours. Therefore, Fig. 4 shows the dependence of the $\mathrm{PH}$ on the concentration of bentonite in dispersive media not carbonated (curve 1) and carbonated (curve 2) after $24 \mathrm{~h}$ of sedimentation.

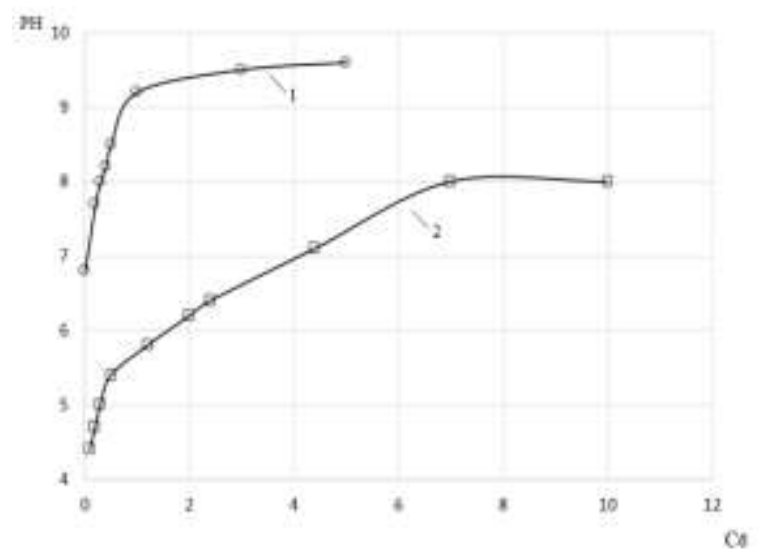

Fig. 4. The dependence of $\mathrm{PH}$ on the concentration of bentonite aqueous suspension of non-carbonated (curve 1) and carbonated (curve 2).

It can be seen that in reference samples without gas, with an increase in the concentration of bentonite, the $\mathrm{pH}$ quickly increases from 6.75 to 9.5 at Sat $>3 \%$. A further increase in the concentration of bentonite in the sample does not change the $\mathrm{pH}$ of the reference (even) samples. The presence of $\mathrm{CO}_{2}$ gas in the dispersion medium lowers the $\mathrm{pH}$ to 4.5 at low concentrations of bentonite. Moreover, the $\mathrm{pH}$ increases with increasing concentration of bentonite in the suspension and takes a constant value at $\mathrm{Sat}>7 \%$.

One may conclude that an increase in the concentration of carbon dioxide in an aqueous suspension of bentonite can decrease the sedimentation time of the solid phase by a factor of ten, reduce the degree of swelling and the $\mathrm{pH}$ value by tens of percent. In this case, an interesting question is the following: How does the chemical composition of bentonite change in samples with $\mathrm{CO} 2$ gas in comparison with the reference ones? It should be noted that many publications are devoted to changes in the chemical composition of bentonite in order to increase its characteristics of adsorption, astringent, colloid, thixotropic, intercolation [7-10].

We have studied the chemical composition of daily precipitation in aqueous suspensions of bentonite of various concentrations with $\mathrm{CO}_{2}$ (odd samples) and even gas into which no gas was introduced. The results are presented in Table I.

TABLE I. CHEMICAL COMPOSITION OF PRECIPITATIONS IN WATER SUSPENSIONS OF BENTONITE IN VARIOUS CONCENTRATIONS

\begin{tabular}{|c|c|c|c|c|c|c|c|c|c|c|}
\hline $\begin{array}{c}\text { Samples } \\
\text { Elements }\end{array}$ & $\mathbf{1}$ & $\mathbf{2}$ & $\mathbf{3}$ & $\mathbf{4}$ & $\mathbf{5}$ & $\mathbf{6}$ & $\mathbf{7}$ & $\mathbf{8}$ & $\mathbf{9}$ & $\mathbf{1 0}$ \\
\hline $\mathrm{C}$ & 0.85 & 0.92 & 0.63 & 0.85 & 0.87 & 0.80 & 0.85 & 0.85 & 0.73 & 0.89 \\
\hline $\mathrm{O}$ & 52.26 & 51.15 & 50.52 & 47.59 & 50.12 & 47.20 & 50.26 & 47.42 & 47.96 & 47.15 \\
\hline $\mathrm{Na}$ & 1.13 & 0.92 & 0.75 & 1.08 & 1.02 & 1.24 & 1.22 & 1.72 & 1.75 & 1.65 \\
\hline $\mathrm{Mg}$ & 1.00 & 0.82 & 0.95 & 1.15 & 0.97 & 0.98 & 0.95 & 1.13 & 0.96 & 1.21 \\
\hline $\mathrm{AI}$ & 8.40 & 7.63 & 9.02 & 9.59 & 9.08 & 9.27 & 8.90 & 9.61 & 9.24 & 9.42 \\
\hline $\mathrm{Si}$ & 26.52 & 29.42 & 26.24 & 28.28 & 26.86 & 29.52 & 26.95 & 28.13 & 27.67 & 28.29 \\
\hline $\mathrm{K}$ & 2.68 & 2.45 & 2.88 & 2.85 & 2.69 & 2.94 & 2.80 & 2.77 & 2.94 & 2.95 \\
\hline $\mathrm{Ca}$ & 1.08 & 1.49 & 1.78 & 1.69 & 1.38 & 1.59 & 1.61 & 1.65 & 1.47 & 1.51 \\
\hline $\mathrm{Ti}$ & 0.72 & 0.67 & 0.80 & 0.78 & 0.76 & 0.59 & 0.64 & 0.62 & 0.80 & 0.69 \\
\hline $\mathrm{Fe}$ & 5.14 & 4.42 & 6.20 & 5.92 & 6.01 & 5.70 & 5.67 & 5.90 & 6.25 & 5.95 \\
\hline $\mathrm{Co}$ & 0.23 & 0.11 & 0.21 & 0.22 & 0.26 & 0.17 & 0.15 & 0.21 & 0.22 & 0.28 \\
\hline
\end{tabular}

As it can be seen from the table above, in all samples, as the value of Sat increases, the concentration of alkali metals $\mathrm{Na}, \mathrm{K}, \mathrm{Ca}, \mathrm{Mg}$ increases noticeably. For example, the sodium concentration increased from 1.13 to $1.75 \%$. This can be explained by a decrease in the solubility of sodium salts under conditions of water deficiency caused by an increase in the concentration of bentonite in suspensions. For the same reason, the growth of the $\mathrm{pH}$ of the dispersion medium slows down as the concentration of bentonite increases. In the interaction of carbon dioxide with alkaline elements, the formation of carbides of different solubility in the dispersion medium occurs. In these reactions, part of the carbon dioxide is bound to bentonite. This leads, with the same saturation, to an increase in the oxygen concentration in all sediments of the solid phase of aerated samples compared to the control samples. It does not exceed 5\%, but indicates the participation of $\mathrm{CO}_{2}$ gas in chemical reactions. It follows from the table that for identical Sat in the solid phase of all odd carbonated samples, the concentration of $\mathrm{Si}$ is lower than in the control ones. At concentrations of bentonite in suspensions up to $2 \%$, the indicated decrease in Si reaches $10 \%$, and at large values of Sat it is about 5\%. It is known that silicon in an amorphous form absorbs a significant amount of various gases; in addition, it is partially converted into a solution even in a weakly alkaline medium. The surface of quartz in the water emits silica in the form of particles of colloidal size. In an aqueous and alkaline medium, silica suspensions form both soluble silica and colloidal crystalline particles smaller than a micrometer $[10,11]$. Therefore, we explain the decrease in the concentration of silicon in samples of bentonite with $\mathrm{CO}_{2}$ gas by the increase in its concentration in the dispersion medium.

\section{CONCLUSION}

For aqueous suspensions of bentonite from Katayama deposit, carbon dioxide can be used as a means of suppressing the degree of swelling, if the concentration of the solid phase is less than $7 \%$. In this process, the $\mathrm{pH}$ of the dispersion medium is significantly reduced, and the elemental composition of the solid phase changes. It was found that in all experimental samples the concentration of silicon in the solid phase decreases to $10 \%$, which is possibly due to the dissolution of its nano-sized particles in a dispersion medium. 


\section{References}

[1] S.V. Krupin, V.A. Trofimova. Colloid-chemical bases for the creation of clay suspensions for industrial work. Kazan, 2010.

[2] Ya.A. Ryazanov. Drilling Fluids Encyclopedia. Orenburg, 2005. p.663.

[3] B.V. Pokidko, M.Yu. Pletnev, M.M. Melnikova, "Influence of electrolyte on the process of structure formation in aqueous dispersions of Na + montmorollonite of the Tagansky field," Vestnik MITHT, vol.6, No. 6, pp. 13-19, 2011.

[4] R.P. Fedorkin, M.G. Khramchenko, "Clay swelling and filtering of solutions in clays," Scientific notes of Kazan State University. Natural Sciences, vol. 152, No. 1. pp. 235-243, 2010.

[5] Sh.A. Gafarov, "Using the product of liquid-phase oxidation of hydrocarbons to stabilize and suppress clay swelling", Oil and gas business, pp.1-5, 2003.
[6] O.S. Trukhina, I.A. Sintsov, "Experience in the Use of Carbon Dioxide for Enhanced Oil Recovery," Advances in Modern Science. No. 3, pp. 205-209, 2016.

[7] A.M. Akimbaeva, E.E. Yergozhin, "Evaluation of the structural and sorption characteristics of activated bentonite," Colloid Journal, vol.69, No. 4, pp. 437-443, 2007.

[8] F.F. Finevich, N.A. Allert, T.R. Karpova, V.K. Duplyakin, "Composite Nanomaterials Based on Acid-Activated Montmorollonites" Ros. Chem. Journals, vol. 1, No. 4, pp. 69-74, 2007.

[9] Benito I. Blanco C., Martinez M. [at al]. J Therm. Anal. No.55, pp. 461, 1999.

[10] A.L. Daudova, V.Kh. Mezhidov, A.M. Abdullaev, M.B. Elmurzaev "Dependence of the size of colloidal silica nanoparticles on the parameters of their synthesis", Nanotechnology engineering journal, No. 3 (35). pp. 30-32, 2013

[11] R.A. Lidin, V.A. Milk, V.A. Andreeva, Inorganic chemistry in reactions. Directory. Moscow: Drofa, pp. 637, 2007. 